OPEN ACCESS

Edited by:

Krishnan Chakravarthy,

University of California, San Diego,

United States

Reviewed by:

Matteo Bologna,

Sapienza University of Rome, Italy

Michael D. Staudt,

Oakland University William Beaumont

School of Medicine, United States

*Correspondence:

Tommaso Bocci

tommaso.bocci@unimi.it

Specialty section:

This article was submitted to

Experimental Therapeutics,

a section of the journal

Frontiers in Neurology

Received: 15 April 2021

Accepted: 30 July 2021

Published: 06 September 2021

Citation:

Guidetti M, Ferrucci R, Vergari $M$,

Aglieco G, Naci A, Versace $S$, Pacheco-Barrios K, Giannoni-Luza S, Barbieri S, Priori A and Bocci T (2021)

Effects of Transcutaneous Spinal Direct Current Stimulation (tsDCS) in Patients With Chronic Pain: A Clinical and Neurophysiological Study.

Front. Neurol. 12:695910.

doi: 10.3389/fneur.2021.695910

\section{Effects of Transcutaneous Spinal Direct Current Stimulation (tsDCS) in Patients With Chronic Pain: A Clinical and Neurophysiological Study}

\author{
Matteo Guidetti ${ }^{1,2}$, Roberta Ferrucci ${ }^{1,3}$, Maurizio Vergari ${ }^{4}$, Giada Aglieco ${ }^{4}$, Anisa Naci ${ }^{4}$, \\ Sara Versace ${ }^{4}$, Kevin Pacheco-Barrios $5,6,7$, Stefano Giannoni-Luza ${ }^{5,6}$, Sergio Barbieri ${ }^{4}$, \\ Alberto Priori ${ }^{1,3}$ and Tommaso Bocci ${ }^{1,3 *}$ \\ 1 "Aldo Ravelli" Center for Neurotechnology and Experimental Brain Therapeutics, Department of Health Sciences, University \\ of Milan, Milan, Italy, ${ }^{2}$ Department of Electronics, Information and Bioengineering, Politecnico di Milano, Milan, Italy, ${ }^{3}$ Azienda \\ Socio-Sanitaria Territoriale-Santi Paolo e Carlo University Hospital, Milan, Italy, ${ }^{4}$ Neurophysiology Unit, Foundation Istituto di \\ Ricerca e Cura a Carattere Scientifico Ca' Granda Ospedale Maggiore Policlinico, Milan, Italy, ${ }^{5}$ Neuromodulation Center, \\ Spaulding Rehabilitation Hospital, Boston, MA, United States, ${ }^{6}$ Center for Clinical Research Learning, Massachusetts \\ General Hospital, Boston, MA, United States, ${ }^{7}$ Unidad de Investigación para la Generación y Síntesis de Evidencias en \\ Salud, Universidad San Ignacio de Loyola, Lima, Peru
}

Background and Aims: Chronic pain is a complex clinical condition, often devastating for patients and unmanageable with pharmacological treatments. Converging evidence suggests that transcutaneous spinal Direct Current Stimulation (tsDCS) might represent a complementary therapy in managing chronic pain. In this randomized, double-blind and sham-controlled crossover study, we assessed tsDCS effects in chronic pain patients.

Methods: Sixteen patients (aged $65.06 \pm 16.16$ years, eight women) with chronic pain of different etiology underwent sham and anodal tsDCS (anode over the tenth thoracic vertebra, cathode over the somatosensory cortical area: $2.5 \mathrm{~mA}, 20 \mathrm{~min}, 5$ days for 1 week). As outcomes, we considered the Visual Analog Scale (VAS), the Neuropathic Pain Symptom Inventory (NPSI), and the components of the lower limb flexion reflex (LLFR), i.e., RIII threshold, RII latency and area, RIII latency and area, and flexion reflex (FR) total area. Assessments were conducted before (TO), immediately at the end of the treatment (T1), after 1 week (T2) and 1 month (T3).

Results: Compared to sham, anodal tsDCS reduced RIII area at T2 $(p=0.0043)$ and T3 $(p=0.0012)$; similarly, FR total area was reduced at T3 $(p=0.03)$. Clinically, anodal tsDCS dampened VAS at T3 $(p=0.015)$, and NPSI scores at T1 $(p=0.0012)$, and T3 $(p=0.0015)$, whereas sham condition left them unchanged. Changes in VAS and NPSI scores linearly correlated with the reduction in LLFR areas $(p=0.0004)$.

Conclusions: Our findings suggest that tsDCS could modulate nociceptive processing and pain perception in chronic pain syndromes.

Keywords: chronic pain, treatment, transcutaneous spinal direct current stimulation, tsDCS, non-invasive brain stimulation, neurophysiology 


\section{INTRODUCTION}

Chronic pain is one of the most intractable clinical problems faced by clinicians and can be devastating for patients (1). Central pain cannot be fully explained based on somatic or neuropathic processes, relying on changes both in ascending and descending nociceptive pathways (2). Different factors seem to contribute to the chronization of pain, both at cortical and spinal level, including functional reorganization of the cortical sensorimotor maps, thalamo-cortical dysrhythmia and spinal "phenotypic switch" in the expression of neuropeptides (3-6). The complexity and heterogeneity of these maladaptive mechanisms make pharmacological treatments often inappropriate.

Several non-invasive brain stimulation (NIBS) techniques have given evidence to improve pain; cerebellar transcranial direct current stimulation (tDCS), a recent technique of stimulation, has shown promising results for pain reduction $(7,8)$, while high-frequency repetitive Transcranial Magnetic Stimulation (rTMS) and anodal tDCS applied over the contralateral primary motor cortex (M1) have been suggested as effective non-pharmacological tools for chronic pain (913). Among NIBS techniques, a growing body of literature indicate that transcutaneous spinal direct current stimulation (tsDCS) modulates both spinal and supra-spinal excitability $(14,15)$. Indeed, in the last decades, different studies applied DC stimulation over the thoracic and cervical spinal cord to modulate spinal pathways (16-18) in humans, providing compelling evidence that tsDCS affects somatosensory, motor, and nociceptive spinal circuits $(14,15,19-21)$. tsDCS changes not only the conductive but also trans-synaptic efficacy of spinal neurons $(21,22)$. Although the mechanisms of action are still to be elucidated, tsDCS likely modulates also the supra-spinal excitability, probably in a polarity-dependent manner $(23,24)$, with anodal tsDCS leading to an impaired intracortical excitability and a functional disconnection between hemispheres $(24,25)$. In this scenario, Cogiamanian et al. (19) found that anodal tsDCS induced lower limb flexion reflex (LLFR) depression in healthy subjects and confirmed that the non-invasive spinal neuromodulatory technique could modulate central nociceptive signal transmission; therefore, it might represent a complementary therapy to drugs and invasive spinal cord stimulation (SCS) in managing chronic pain.

LLFR is a polysynaptic and multi-segmental spinal reflex that induces a complex flexion synergy of the stimulated limb; it has two main components, formally named RII and RIII, which derive from the activation of the cutaneous A-beta and nociceptive A-delta fibers, respectively (26-28). Guidelines recommend the RIII reflex as the most reliable nociceptive reflex for the assessment of pain treatment efficacy (29); it has also been proposed as a neural window onto the spinal mechanisms activated during locomotion, both in animals (30-32) and in humans (28).

To the best of our knowledge, no study has evaluated to date whether tsDCS modulates the RIII reflex, as a measure of the central nociceptive processing in chronic pain patients. Our aim was to evaluate the use of tsDCS, applied over the spinal cord (spinous process of the tenth thoracic vertebra, the anode) and primary somatosensory cortex (S1, the cathode), as non-pharmacological treatment in patients with chronic pain of different etiologies, by monitoring the effects on the LLFR and clinical scales, as assessed by the Visual Analog Scale (VAS) and the Neuropathic Pain Symptom Inventory (NPSI).

\section{MATERIALS AND METHODS}

\section{Subjects}

Sixteen patients (aged $65.06 \pm 16.16$ years, 8 women) with chronic pain have been enrolled in our study. Demographic features are summarized in Table 1. Inclusion criteria were: (1) age 18-70 years; (2) normal score ( $>24)$ at the Mini-Mental State Examination; (3) stable presence of pain for at least 5 years, (4) no coexistence of major neurologic, neuropsychological, and psychiatric diseases as confirmed by clinical history and anamnestic interview; and (5) stable pharmacological therapy during the month before the inclusion. Exclusion criteria were as follows: (1) participants who started new medical treatments or physiotherapy within 1 month prior of the recruitment; (2) participants had any history/current signs or symptoms of neurologic or psychiatric disorders; (3) deep brain stimulation implanted patients; (4) pregnancy; and (5) carriers of spinal cord stimulators. The study protocol followed to the Declaration of Helsinki and was approved by the Ethics Committee of the IRCCS Ca' Granda Foundation-Maggiore Policlinico Hospital of Milan. All subjects gave written informed consents before the participation.

\section{Study Design and Experimental Procedures}

In this randomized, double-blind, sham-controlled crossover study, each patient underwent sham and anodal tsDCS $(2.5 \mathrm{~mA}$ for $20 \mathrm{~min}$, once a day for 5 days). Participants were assigned randomly to either group A (active tsDCS first) or group B (sham tsDCS first), using a random number generator. At the next treatment, patients were crossed over to the opposite treatment. Due to the potential carryover effect from crossover design, a washout period of at least 4 weeks was provided between treatments. Clinical (VAS and NPSI) and neurophysiological (LLFR) outcome were assessed before (T0) and at the end (T1) of the 5-day treatment and 1 week (T2) and 1 month (T3) after the completion of the stimulation protocol (see Figure 1). Both subjects and assessors were blinded to the stimulation condition.

\section{Transcutaneous Spinal Direct Current Stimulation}

The application of spinal DCS followed key technical features already verified in literature $(14,19,33,34)$. Briefly, patients were asked to lie down on a couch, and tsDCS (2.5 mA, $20 \mathrm{~min})$ was delivered by a programmable stimulator (HDCStim ${ }^{\mathrm{TM}}$, Newronika, Italy) connected to a pair of rectangular electrodes; the anode was placed over the spinous process of the tenth thoracic vertebra (from the 10th to 12th vertebra, with the major axis parallel to spinal cord), and the cathode over the somatosensory cortical area ( $2 \mathrm{~cm}$ rear of CZ, according to $10 / 20$ EEG system). The tsDCS electrodes were rectangular pieces of 
TABLE 1 | Demographic characteristics of the subjects.

\begin{tabular}{|c|c|c|c|c|c|}
\hline Patient no. & Etiology of pain & Timeline of intervention & Pharmacological treatment & Baseline VAS & Baseline NPSI \\
\hline 1 & Post-herpes & Anodal/Sham & Pregabalin & $71 / 76$ & $41 / 51$ \\
\hline 2 & Lumbosacral Radiculopathy & Sham/Anodal & Oxycodone/naloxone & $63 / 45$ & $13 / 19$ \\
\hline 3 & Lumbosacral Radiculopathy & Anodal/Sham & None & $57 / 52$ & $67 / 40$ \\
\hline 4 & Idiopathic & Anodal/Sham & Methylprednisolone & $89 / 90$ & $17 / 22$ \\
\hline 5 & Diabetes & Sham/Anodal & None & $23 / 61$ & $12 / 36$ \\
\hline 6 & Lumbosacral Radiculopathy & Anodal/Sham & Etoricoxib & $98 / 61$ & $61 / 36$ \\
\hline 7 & Lumbosacral Radiculopathy & Sham/Anodal & Clonazepam & $34 / 61$ & $43 / 36$ \\
\hline 8 & Lumbosacral Radiculopathy & Anodal/Sham & None & $75 / 21$ & $40 / 12$ \\
\hline 9 & Lumbosacral Radiculopathy & Anodal/Sham & None & $44 / 28$ & $48 / 42$ \\
\hline 10 & Lumbosacral Radiculopathy & Anodal/Sham & None & $100 / 64$ & $47 / 40$ \\
\hline 11 & Multiple Sclerosis & Sham/Anodal & Etoricoxib and Baclofen & $100 / 69$ & $81 / 70$ \\
\hline 12 & Lumbosacral Radiculopathy & Anodal/Sham & None & $70 / 85$ & $56 / 34$ \\
\hline 13 & Diabetes & Sham/Anodal & None & $40 / 61$ & $44 / 36$ \\
\hline 14 & PLMT & Sham/Anodal & Levetiracetam & 95/97 & $58 / 57$ \\
\hline 15 & Lumbosacral Radiculopathy & Sham/Anodal & Oxycodone/naloxone & $36 / 40$ & $20 / 13$ \\
\hline 16 & Post-herpes & Sham/Anodal & Pregabalin & $66 / 61$ & $43 / 36$ \\
\hline
\end{tabular}

PLMT, painful legs and moving toes syndrome; VAS, Visual Analog Scale; NPSI, Neuropathic Pain Symptom Inventory.

saline-soaked synthetic sponge $\left(7 \times 8 \mathrm{~cm}, 56 \mathrm{~cm}^{2}\right)$. We applied a current density of $0.035 \mathrm{~mA} / \mathrm{cm}^{2}$ and delivered a total charge density of $42.8 \mathrm{mC} / \mathrm{cm}^{2}$, below the threshold values reported for tissue damage $(35,36)$. At the onset of tsDCS, the current was increased for $30 \mathrm{~s}$, and at the offset, it was decreased for $30 \mathrm{~s}$ in a ramp-like manner, a method shown to achieve a good level of blinding among sessions $(37,38)$. For a sham tsDCS, the current was turned on for $5 \mathrm{~s}$ and then turned off in a rampshaped fashion, thus inducing skin sensations indistinguishable from anodal tsDCS. Assessors and patients were blinded to the tsDCS protocol; patients did not discriminate between anodal and sham condition, neither reported adverse effects as evidenced by the questionnaire developed by Brunoni et al., which was administered to each patient (12).

\section{LLFR Recording}

Lower limb flexion reflex (LLFR) was recorded from the left lower limb with patients in a prone position on a bed in a prepared room, with the ankle flexed at $90^{\circ}$. $\mathrm{Ag} / \mathrm{AgCl}$ surface-capping electrodes placed with patches were used to guarantee the signal was recorded at the same position during all the experiments and ensure a reliable registration. Furthermore, an electro-conductive gel was used under the stimulating electrodes to optimize the passage of the currents and increase patient compliance. The RIII reflex was evoked and recorded from the left lower limb, as described in detail elsewhere $(39,40)$. Briefly, the recording electrode was placed on the short head of the biceps femoris; the reference electrode was placed on the capital of the fibula; and the earth electrode was placed on gastrocnemius muscle, in an intermediate position between the stimulation and registration site. The stimulation was percutaneous and ipsilateral to the recording side; the electrodes were placed at the retro-malleolar pathway of the sural nerve. Five responses were recorded after a stimulus of $1 \mathrm{~ms}$ duration, elapsed by $5-15$ interstimulus intervals
(ISI) to avoid habituation $(27,41)$. The signal was subsequently corrected and averaged, and the following parameters were quantified: the RIII threshold ( $\mathrm{mA})$, i.e., the minimum current intensity at which the reflection appears, the latencies (ms), and the area $\left(\mathrm{mV}^{*} \mathrm{~ms}\right)$ of the main components of the $\mathrm{FR}$ (RII and RIII) were assessed. Stimuli with increased intensities were delivered with an interval of $2.5 \mathrm{~mA}$ at a time to obtain a repeatable response in three consecutive recordings, with a minimum width of at least $50 \mu \mathrm{V}$. The RII response, originated from the sensory activation of the nerve fibers, usually appeared with a lower stimulus intensity compared to RIII, with latency between 40 and $80 \mathrm{~ms}$. Once the RII response was obtained, the intensity of the stimulus was increased until the RIII response appeared. Five responses of FR were recorded with a stimulus intensity set at the threshold $20 \%$ higher than RIII. Since the FR is variable, the recording was kept in a constant time of day for all the evaluation time points (T0, T1, T2, and T3). The voluntary EMG activity at rest was monitored by video-audio feedback to ensure the lack of significant muscle contraction during experimental sessions.

\section{Clinical Evaluation}

The Visual Analog Scale (VAS) (42) and Neuropathic Pain Symptom Inventory (NPSI) (43) were performed at T0, T1, T2, and T3. The VAS is represented by a $10-\mathrm{cm}$-long line in which one end indicates the absence of pain (0), and the other end corresponds to the worst pain perceived (10). Each patient was asked to indicate the intensity of his/her level of pain with a line (variable between 0 and 10) at that precise moment. NPSI consists of 12 questions to investigate the different types of pain that a patient feels with a score between 0 and 10 (except for two questions in which the maximum assignable score is 5). The final score was the sum of each question and ranges from zero to one hundred. 


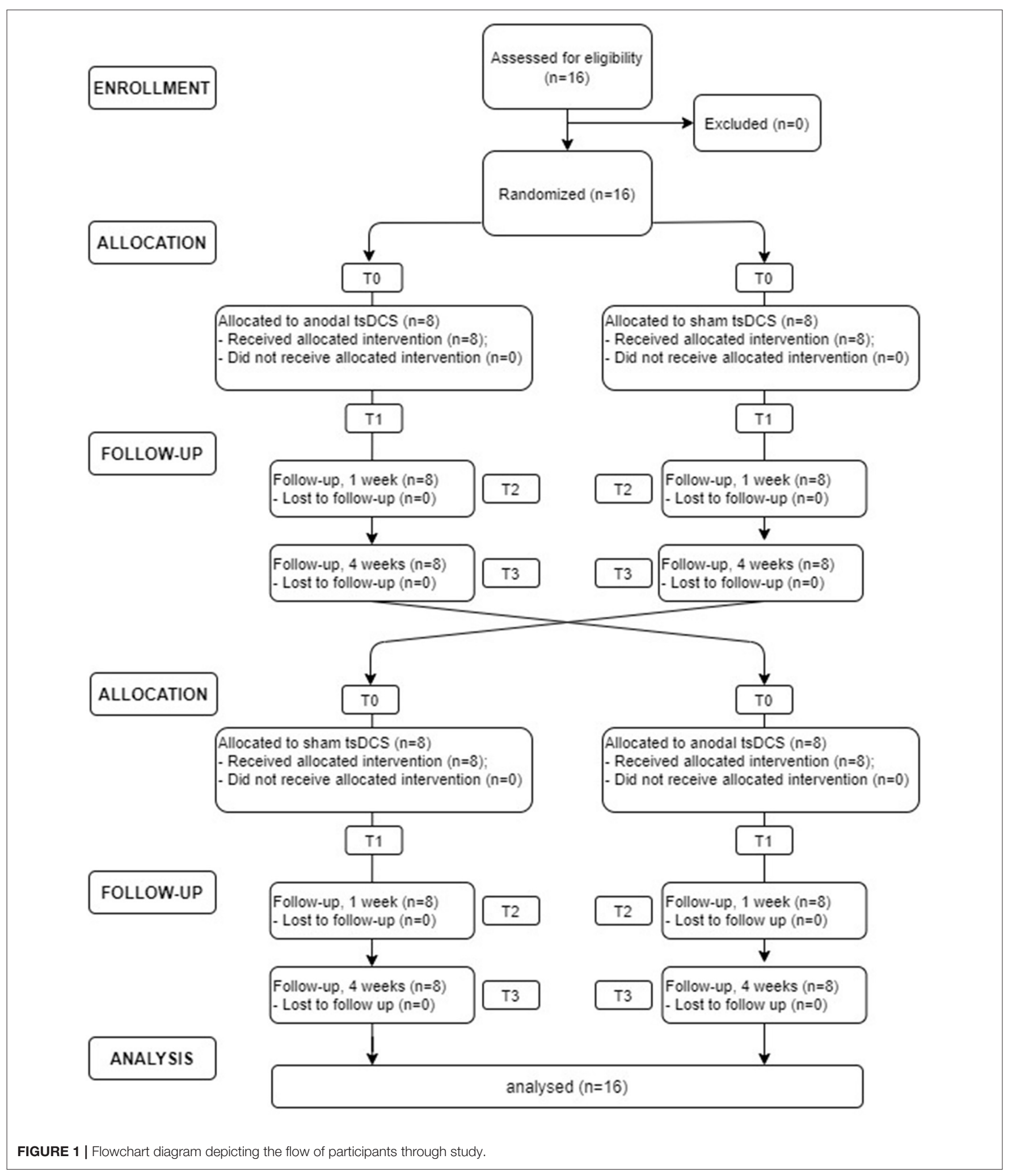

\section{Statistical Analysis}

Non-parametric analyses were used, as all data sets did not pass the Shapiro-Wilk test for normality ( $p<0.05$ ); Mann-Whitney test for each variable was performed on raw data to exclude significant differences at baseline (sham vs. active tsDCS). tsDCSinduced changes in each variable were then assessed by using 
TABLE 2 | Neurophysiological results.

\begin{tabular}{|c|c|c|c|c|c|c|c|c|}
\hline & \multicolumn{4}{|c|}{ Anodal tsDCS } & \multicolumn{4}{|c|}{ Sham tsDCS } \\
\hline RII latency & $77.28 \pm 2.97$ & $83.38 \pm 3.48$ & $83.88 \pm 3.81$ & $80.05 \pm 4.04$ & $65.72 \pm 6.43$ & $69.37 \pm 6.27$ & $70.72 \pm 6.39$ & $70.04 \pm 7.34$ \\
\hline RIII latency & $142.52 \pm 6.10$ & $142.38 \pm 7.34$ & $135.32 \pm 5.47$ & $135.08 \pm 5.05$ & $146.48 \pm 6.18$ & $137.87 \pm 4.35$ & $144.11 \pm 5.54$ & $147.69 \pm 5.29$ \\
\hline RIII area & $5.96 \pm 1.40$ & $5.40 \pm 1.25$ & $4.18 \pm 1.41$ & $4.51 \pm 1.04$ & $4.71 \pm 0.94$ & $6.35 \pm 1.95$ & $7.29 \pm 1.61$ & $6.39 \pm 1.41$ \\
\hline
\end{tabular}

RIII Threshold, RII Latency, RIII latency, RIII area, and FR total area are reported at TO and over time, according to the group (anodal or sham tsDCS). Values are expressed in $\mu V$, as normalized to TO mean values \pm standard error (S.E).

the Friedman test (non-parametric analysis on paired data) with the main factor "time" (four levels: T0, T1, T2, and T3). In order to disclose significant changes at each time point between anodal and sham tsDCS, a Wilcoxon matched-pairs signed test was then applied. Both the electrophysiological measures and clinical scores were normalized to T0 score before entering the analysis [according to the formula (T1 - T0)/T0 * $100+100$ ]. Finally, the Spearman's rank correlation coefficient was used to compare changes in electrophysiological parameters (LLFR) with the clinical outcome. Statistical significance was set at $p<0.05$. The data were analyzed using SPSS v. 21.0 for Windows (SPSS Inc.). Neurophysiological and clinical data are expressed as mean \pm Standard Error (S.E.).

\section{RESULTS}

\section{Neurophysiological Assessment}

Mann-Whitney test did not disclose any significant difference between the two groups at baseline, for each variable assessed $(p>0.05)$. The statistical analysis disclosed that changes both in RIII and FR total area between the two groups were found over time, with anodal tsDCS leading to a marked reduction of both parameters and sham condition eliciting opposite effects (Friedman's test: $p<0.0001$ for RIII and $p=0.027$ for FR total area) (Table 2; Figure 2). Regarding RIII area, this reduction was observed at T2 (Wilcoxon's test, as post-hoc analysis: $p=0.0043$ ) and T3 $(p=0.0012)$; FR total area significantly decreased in the anodal compared to sham tsDCS at T3 (Wilcoxon's test: $p$ $=0.03)$. The sham group showed a significant increase in RII latency from T0 to T3 (Friedman test: $p<0.0001$; T0 vs. T3: $p$ $<0.0001$ ) and in RIII area from T0 to T2 (Friedman test: $p<$ 0.01 ; T0 vs. T2: $p<0.01$; Table 2; Figure 2).

\section{Clinical Outcome}

Mann-Whitney test for each raw clinical variable (sham vs. anodal tsDCS) at baseline revealed no statistical difference between the two groups $(p>0.05)$. The active group significantly changed over time in VAS (Friedman test: $p<0.05$ ) and NPSI (Friedman test: $p<0.0001$ ) scores, with post-hoc pairwise comparison showing a significant reduction for NPSI from T0 to T1 $(p<0.0001)$ and from T0 to T2 $(p<0.0001)$. In sham condition, VAS showed significant changes over time, with scores increasing from T2 to T3 (Friedman test: $p<0.01$; T2 vs. T3: $p<0.01$ ), paralleled by a worsening of NPSI scores (Friedman test: $p<0.01$; T2 vs. T3: $p<0.01$ ) (Table 3; Figure 3). When compared the two stimulation conditions at each time point, VAS was significantly dampened at T3 ( $p=0.015$, Wilcoxon's), whereas NPSI scores improved both at T1 $(p=0.0012)$ and T3 $(p=0.0015)$. Changes in VAS and NPSI scores linearly correlated with the reduction in LLFR areas. Indeed, patients with greater clinical improvement showed a more robust modulation of neurophysiological responses $(p=0.0004$, Spearman's rank correlation coefficient).

\section{DISCUSSION}

Our results demonstrated that the use of tsDCS for chronic pain subjects is safe and feasible. All subjects tolerated the stimulation well without dropouts. Anodal tsDCS dampened both RIII and FR total area and improved NPSI scores over time, thus resulting in an overall analgesic effect.

Anodal tsDCS modulates the nociceptive component (RIII) of the LLFR. These findings are in line with the results reported by Cogiamanian et al. (19), showing a significant reduction of RIII area by $\sim 27 \%$ after anodal thoracic tsDCS in healthy subjects. In our study, we found a range of RIII area reduction higher than in healthy subjects. The RIII reflex is considered as the most reliable nociceptive reflex to assess the efficacy of pain treatments $(27,44)$, and it has been shown to be consistently hyperexcitable in some chronic pain conditions, including fibromyalgia (45, 46), Wallenberg's Syndrome (47), and some types of chronic headaches (48-50).

Moreover, it is important to note the high variability in our estimates (expressed by large standard errors). This is partially explained by our small sample size, and also due to the mixed etiologies of chronic pain we included. The overall neurophysiological results reported on neuropathic and chronic pain tend to be heterogeneous, which might suggest RIII changes to be pathophysiology dependent (27). Another important source of heterogeneity might come from the different pharmacological therapies. Results from tDCS studies clearly suggest that several pharmacological classes interfere with neuromodulation with a complex non-linear interaction $(51,52)$. Therefore, medications may tarnish tDCS effects themselves (51). For example, GABA modulators (e.g., Lorazepam) induced a delayed but prolonged increase of the excitability after 

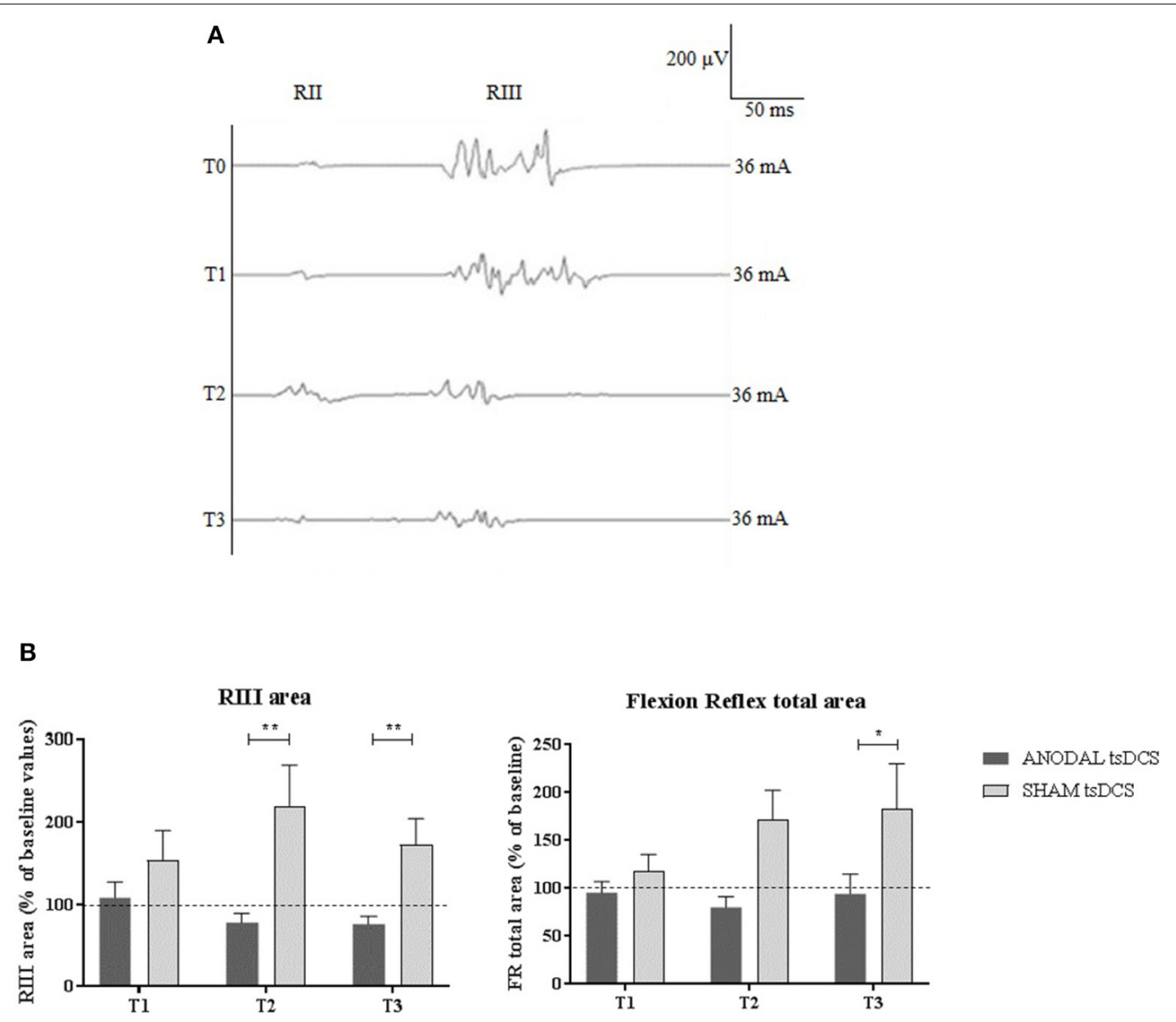

FIGURE 2 | LLFR changes. (A) Exemplificative averaged traces of RIII reflex recorded before (T0), at the end of 5 days treatment (T1), and 1 week (T2) and 1 month (T3) after the completion of anodal tsDCS protocol. (B) Changes in RIII area and FR total area between anodal (black bars) and sham (gray bars) conditions. Data are given as normalized to T0 mean \pm SE. The statistical significance refers to the comparison between anodal (active) and sham (placebo) stimulation $\left({ }^{*} p<0.05\right.$; ${ }^{* *} p<$ 0.001 , Wilcoxon's test post-hoc analysis).

TABLE 3 | Clinical assessment.

\begin{tabular}{|c|c|c|c|c|c|c|c|c|}
\hline & \multicolumn{4}{|c|}{ Anodal tsDCS } & \multicolumn{4}{|c|}{ Sham tsDCS } \\
\hline & TO & T1 & T2 & T3 & TO & T1 & T2 & T3 \\
\hline VAS & $66.33 \pm 6.85$ & $41.40 \pm 9.07$ & $48.13 \pm 7.6$ & $41.75 \pm 9.72$ & $60.64 \pm 7.67$ & $57.82 \pm 9.75$ & $50.10 \pm 10.62$ & $63.80 \pm 7.91$ \\
\hline NPSI & $43.20 \pm 5.28$ & $27.07 \pm 5$ & $29.2 \pm 5.58$ & $34.25 \pm 7.51$ & $36.36 \pm 5.63$ & $36.27 \pm 7.09$ & $32.20 \pm 6.38$ & $41.43 \pm 7.94$ \\
\hline
\end{tabular}

VAS and NPSI scores for anodal or sham tsDCS over time. Values are expressed in $\mathrm{mm}$, as mean values \pm standard error (S.E).

anodal tDCS (53), while glutamatergic antagonist agents (e.g., dextromethorphan) abolish the after-effects of both anodal and cathodal tDCS $(54,55)$.

It has been suggested that RIII reflex variables are correlated with pain-related measurements. For instance, the threshold of the RIII reflex has been shown to correspond to the pain threshold and the area of the reflex to be related to the level of pain perception $(27,56)$. Our results support this hypothesis, since we found a pain intensity reduction in patients with small RIII area in the anodal condition. The within-group pain reduction was higher and consistent in the anodal tsDCS condition with a range of reduction from -18.2 to $-24.58 \mathrm{~mm}$ in VAS, which are considered around the minimum change reflecting clinical differences in pain outcome (57). However, the difference between stimulations was not always significant after multiple comparison adjustment. This could be explained by different factors, such as the small sample size, the short duration of the stimulation protocol (5 days), or the heterogeneity of pain etiologies. Indeed, the need of a protocol optimization to identify the most effective tsDCS stimulation scheme for chronic pain 


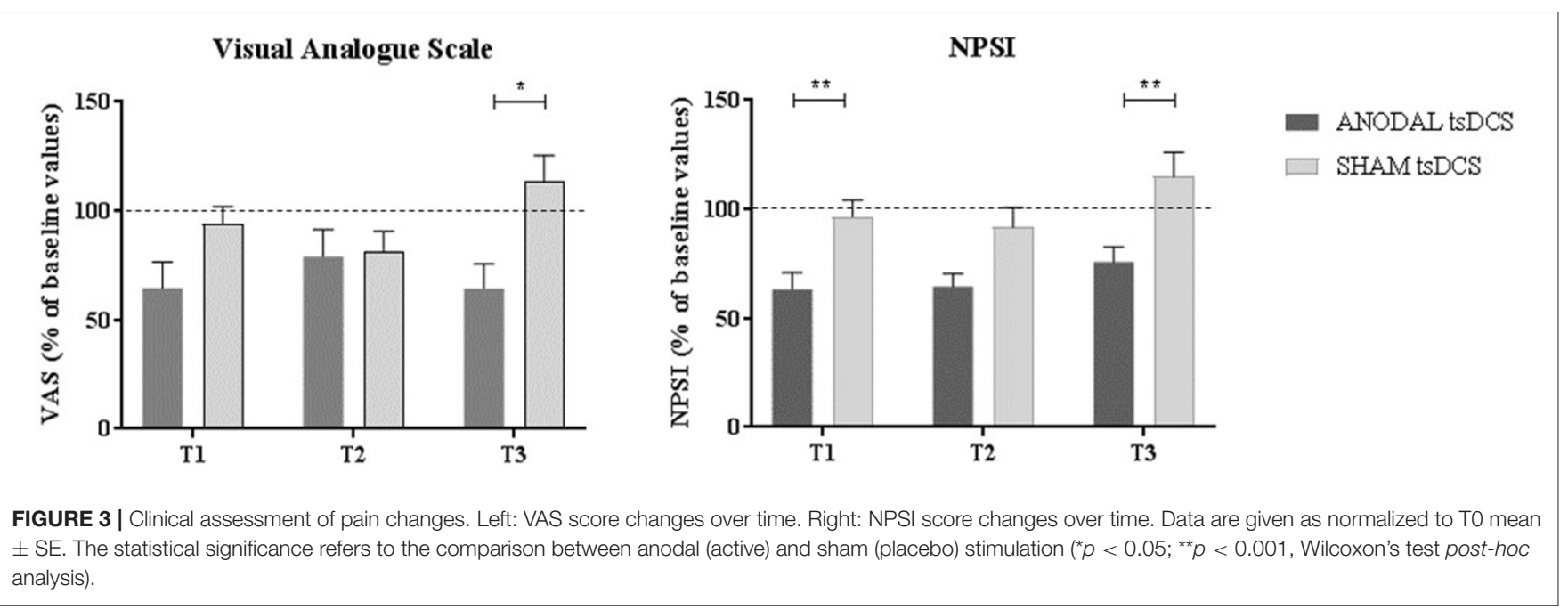

(58) has been reported, considering also the different degrees of central sensitization (59) involved in such condition.

Finally, the lack of changes in RII parameters is not surprising, as this component reflects the activation of non-nociceptive, large-diameter fibers, although recent studies have suggested that $A \beta$ terminals are also engaged in the pathophysiology of paroxysmal pain $(60,61)$.

The pain modulation mechanism of tsDCS remains open to questions. It has been suggested that anodal spinal tsDCS may produce a combination of spinal and supraspinal effects (62). Furthermore, anodal tsDCS can reduce the pain sensitivity associated with nociceptive mechanical stimuli (63) and can modulate temporal summation of pain modulating the activity of wide-dynamic-range neurons (64). Thus, tsDCS could modulate neuronal activity in lemniscal, spinothalamic, and segmental spinal circuits, suggesting glutamatergic, GABAergic, and glycinergic networks involvement (17). Then, tsDCS may ultimately interfere with supra-spinal mechanisms of pain sensitization, comprising the thalamo-cortical dysrhythmia and the maladaptive reorganization of cortical sensorimotor maps. Considering these potential mechanisms of action, we selected the position of the reference electrode in the somatosensory cortex. Parazzini et al. (65) estimated, based on current density simulation, that a cortical reference (attached to $\mathrm{Cz}$ ) produces a higher magnitude of the current density in the cervical and thoracic regions, and it could improve its physiological effects by the site of action at both the spinal and supraspinal levels. Assuming the expected pain-related maladaptive plasticity in spinal and supraspinal levels, a montage with a cortical reference electrode was applied to maximize the neuroplastic effects of tsDCS in this study.

Moreover, Truini et al. (15) showed a significant decrease of N1 and N2 amplitude of laser evoked potentials (LEP) over the foot, but not over the perioral region after anodal tsDCS over T10. In line with these results, Lenoir et al. (66) reported significant effects of anodal T10 tsDCS over SEP-N2 wave elicited by nociceptive LEP over the foot, but not over the hand nor by non-nociceptive stimuli, suggesting that tsDCS modulatory effects are basically local and influence only the conduction of pain-stimulus coming below the stimulation site. Based on these, it is reasonable to consider the potential influences of the spinal cord injury locations in the design of future studies, as cervical or upper thoracic lesions might not respond the same way as lower thoracic lesions or lesions near the stimulation site.

This study has some limitations. First, the small sample size might have underpowered the study, even though we found neurophysiological and clinically significant results. Second, we included a heterogenous chronic pain population, with multiple neuropathic pain etiologies. The different degree of central nociceptive signal transmission affectation underlying such conditions might have affected the results and reduced the external validity of our findings. Similarly, our patients had stable but different (or even no) pharmacological therapies in place during the study. The different biochemical effects induced by the medications may have affected tsDCS effects, as previously suggested $(51,52)$.

In conclusion, our findings on anodal tsDCS-induced reduction of nociceptive reflex (RIII reflex area) and pain intensity (although marginally significant) in chronic pain subjects confirm that a non-invasive spinal neuromodulatory technique could modulate central nociceptive signal transmission and pain perception. Thus, tsDCS represents a promising intervention to reduce chronic pain by targeting painmaladaptive plasticity. Further studies are needed with larger sample sizes, including a more homogenous population, and with optimized study design (double-blinded, parallel design) and stimulation protocols (longer stimulation protocols, concomitant interventions, spinal, and supraspinal biomarkers).

\section{DATA AVAILABILITY STATEMENT}

The raw data supporting the conclusions of this article will be made available by the authors, without undue reservation. 


\section{ETHICS STATEMENT}

The studies involving human participants were reviewed and approved by Ethics Committee of the IRCCS Ca' Granda Foundation - Maggiore Policlinico Hospital of Milan. The patients/participants provided their written informed consent to participate in this study.

\section{REFERENCES}

1. Mills SEE, Nicolson KP, Smith BH. Chronic pain: a review of its epidemiology and associated factors in population-based studies. Br J Anaesth. (2019) 123:e273-83. doi: 10.1016/j.bja.2019.03.023

2. Yarnitsky D. Role of endogenous pain modulation in chronic pain mechanisms and treatment. Pain. (2015) 156:S2431. doi: 10.1097/01.j.pain.0000460343.46847.58

3. Suzuki R, Dickenson A. Spinal and supraspinal contributions to central sensitization in peripheral neuropathy. Neurosignals. (2005) 14:175-81. doi: 10.1159/000087656

4. Drolet M, Brisson M, Schmader K, Levin M, Johnson R, Oxman M, et al. Predictors of postherpetic neuralgia among patients with herpes zoster: a prospective study. J Pain. (2010) 11:1211-21. doi: 10.1016/j.jpain.2010.02.020

5. Flor H. Phantom-limb pain: characteristics, causes, and treatment. Lancet Neurol. (2002) 1:182-9. doi: 10.1016/s1474-4422(02)00074-1

6. Alshelh Z, di Pietro F, Youssef AM, Reeves JM, Macey PM, Russell Vickers E, et al. Chronic neuropathic pain: it's about the rhythm. J Neurosci. (2016) 36:1008-18. doi: 10.1523/JNEUROSCI.2768-15.2016

7. Bocci T, De Carolis G, Ferrucci R, Paroli M, Mansani F, Priori A, et al. Cerebellar transcranial direct current stimulation (ctDCS) ameliorates phantom limb pain and non-painful phantom limb sensations. Cerebellum. (2019) 18:527-35. doi: 10.1007/s12311-019-01020-w

8. Bocci T, Santarcangelo E, Vannini B, Torzini A, Carli G, Ferrucci R, et al. Cerebellar direct current stimulation modulates pain perception in humans. Restor Neurol Neurosci. (2015) 33:597-609. doi: 10.3233/RNN-140453

9. Fregni F, El-Hagrassy MM, Pacheco-Barrios K, Carvalho S, Leite J, Simis $\mathrm{M}$, et al. Evidence-based guidelines and secondary meta-analysis for the use of transcranial direct current stimulation (tDCS) in neurological and psychiatric disorders. Int J Neuropsychopharmacol. (2021) 24:256313. doi: 10.1093/ijnp/pyaa051

10. Fregni F, Gimenes R, Valle AC, Ferreira MJL, Rocha RR, Natalle L, et al. A randomized, sham-controlled, proof of principle study of transcranial direct current stimulation for the treatment of pain in fibromyalgia. Arthritis Rheum. (2006) 54:3988-98. doi: 10.1002/art.22195

11. Antal A, Terney D, Kühnl S, Paulus W. Anodal transcranial direct current stimulation of the motor cortex ameliorates chronic pain and reduces short intracortical inhibition. J Pain Symptom Manage. (2010) 39:890903. doi: 10.1016/j.jpainsymman.2009.09.023

12. Brunoni AR, Amadera J, Berbel B, Volz MS, Rizzerio BG, Fregni F. A systematic review on reporting and assessment of adverse effects associated with transcranial direct current stimulation. Int J Neuropsychopharmacol. (2011) 14:1133-45. doi: 10.1017/S1461145710001690

13. Lefaucheur JP, André-Obadia N, Antal A, Ayache SS, Baeken C, Benninger $\mathrm{DH}$, et al. Evidence-based guidelines on the therapeutic use of repetitive transcranial magnetic stimulation (rTMS). Clin Neurophysiol. (2014) 125:2150-206. doi: 10.1016/j.clinph.2014.05.021

14. Cogiamanian F, Vergari M, Pulecchi F, Marceglia S, Priori A. Effect of spinal transcutaneous direct current stimulation on somatosensory evoked potentials in humans. Clin Neurophysiol. (2008) 119:2636-40. doi: 10.1016/j.clinph.2008.07.249

15. Truini A, Vergari M, Biasiotta A, La Cesa S, Gabriele M, Di Stefano G, et al. Transcutaneous spinal direct current stimulation inhibits nociceptive spinal pathway conduction and increases pain tolerance in humans. Eur J Pain. (2011) 15:1023-7. doi: 10.1016/j.ejpain.2011.04.009

16. Ahmed Z. Trans-spinal direct current stimulation modulates motor cortex-induced muscle contraction in mice. $J$ Appl

\section{AUTHOR CONTRIBUTIONS}

MV and SB: concept and design. MV, GA, AN, and SV: acquisition of data. MV and TB: interpretation of data. MG, RF, KP-B, SG-L, and TB: drafting of the manuscript. AP: critical revision of the manuscript. All authors contributed to the article and approved the submitted version.

\section{Physiol. (2011) 110:1414-24. doi: 10.1152/japplphysiol.0139} 0.2010

17. Ahmed Z, Wieraszko A. Trans-spinal direct current enhances corticospinal output and stimulation-evoked release of glutamate analog, D-2,3- ${ }^{3}$ H-aspartic acid. J Appl Physiol. (2012) 112:157692. doi: 10.1152/japplphysiol.00967.2011

18. Aguilar J, Pulecchi F, Dilena R, Oliviero A, Priori A, Foffani G. Spinal direct current stimulation modulates the activity of gracile nucleus and primary somatosensory cortex in anaesthetized rats. J Physiol. (2011) 589:498196. doi: 10.1113/jphysiol.2011.214189

19. Cogiamanian F, Vergari M, Schiaffi E, Marceglia S, Ardolino G, Barbieri $S$, et al. Transcutaneous spinal cord direct current stimulation inhibits the lower limb nociceptive flexion reflex in human beings. Pain. (2011) 152:3705. doi: 10.1016/j.pain.2010.10.041

20. Lamy J-CC, Ho C, Badel A, Arrigo RT, Boakye M. Modulation of soleus H reflex by spinal DC stimulation in humans. J Neurophysiol. (2012) 108:90614. doi: $10.1152 /$ jn. 10898.2011

21. Winkler T, Hering P, Straube A. Spinal DC stimulation in humans modulates post-activation depression of the $\mathrm{H}$-reflex depending on current polarity. Clin Neurophysiol. (2010) 121:957-61. doi: 10.1016/j.clinph.2010.01.014

22. Bocci T, Vannini B, Torzini A, Mazzatenta A, Vergari M, Cogiamanian F, et al. Cathodal transcutaneous spinal direct current stimulation (tsDCS) improves motor unit recruitment in healthy subjects. Neurosci Lett. (2014) 578:75-9. doi: 10.1016/j.neulet.2014.06.037

23. Bocci T, Marceglia S, Vergari M, Cognetto V, Cogiamanian F, Sartucci F, et al. Transcutaneous spinal direct current stimulation modulates human corticospinal system excitability. J Neurophysiol. (2015) 114:4406. doi: 10.1152/jn.00490.2014

24. Bocci T, Barloscio D, Vergari M, Di Rollo A, Rossi S, Priori A, et al. Spinal direct current stimulation modulates short intracortical inhibition. Neuromodul Technol Neural Interface. (2015) 18:686-93. doi: 10.1111/ner.12298

25. Bocci T, Caleo M, Vannini B, Vergari M, Cogiamanian F, Rossi $S$, et al. An unexpected target of spinal direct current stimulation: interhemispheric connectivity in humans. J Neurosci Methods. (2015) 254:18-26. doi: 10.1016/j.jneumeth.2015.07.012

26. Willer JC, Bathien N. Pharmacological modulations on the nociceptive flexion reflex in man. Pain. (1977) 3:111-9. doi: 10.1016/0304-3959(77)90074-4

27. Sandrini G, Serrao M, Rossi P, Romaniello A, Cruccu G, Willer JC. The lower limb flexion reflex in humans. Prog Neurobiol. (2005) 77:35395. doi: 10.1016/j.pneurobio.2005.11.003

28. Ertekin C, Ertekin N, Karcioglu M. Conduction velocity along human nociceptive reflex afferent nerve fibres. J Neurol Neurosurg Psychiatry. (1975) 38:959-65. doi: 10.1136/jnnp.38.10.959

29. Cruccu G, Sommer C, Anand P, Attal N, Baron R, Garcia-Larrea $\mathrm{L}$, et al. EFNS guidelines on neuropathic pain assessment: Revised (2009). Eur J Neurol. (2010) 17:1010-8. doi: 10.1111/j.1468-1331.2010.0 2969.x

30. Lundberg A. Multisensory control of spinal reflex pathways. Prog Brain Res. (1979) 50:11-28. doi: 10.1016/S0079-6123(08)60803-1

31. Schomburg ED, Petersen N, Barajon I, Hultborn H. Flexor reflex afferents reset the step cycle during fictive locomotion in the cat. Exp Brain Res. (1998) 122:339-50. doi: 10.1007/s002210050522

32. Jankowska E, Jukes MGM, Lund S, Lundberg A. The effect of DOPA on the Spinal Cord 5. Reciprocal organization of pathways transmitting excitatory action to alpha motoneurones of flexors and extensors. Acta Physiol Scand. (1967) 70:369-88. 
33. Lamy JC, Boakye M. Seeking significance for transcutaneous spinal DC stimulation. Clin Neurophysiol. (2013) 124:104950. doi: 10.1016/j.clinph.2013.01.007

34. Lim CY, Shin HI. Noninvasive DC stimulation on neck changes MEP. Neuroreport. (2011) 22:819-23. doi: 10.1097/WNR.0b013e32834b939d

35. Iyer MB, Mattu U, Grafman J, Lomarev M, Sato S, Wassermann EM. Safety and cognitive effect of frontal DC brain polarization in healthy individuals. Neurology. (2005) 64:872-5. doi: 10.1212/01.WNL.0000152986.07469.E9

36. Nitsche MA, Liebetanz D, Lang N, Antal A, Tergau F, Paulus W, et al. Safety criteria for transcranial direct current stimulation (tDCS) in humans [1] (multiple letters). Clin Neurophysiol. (2003) 114:22202. doi: 10.1016/s1388-2457(03)00235-9

37. Galea JM, Jayaram G, Ajagbe L, Celnik P. Modulation of cerebellar excitability by polarity-specific noninvasive direct current stimulation. J Neurosci. (2009) 29:9115-22. doi: 10.1523/JNEUROSCI.2184-09.2009

38. Gandiga PC, Hummel FC, Cohen LG. Transcranial DC stimulation (tDCS): a tool for double-blind sham-controlled clinical studies in brain stimulation. Clin Neurophysiol. (2006) 117:845-50. doi: 10.1016/j.clinph.2005.12.003

39. Willer JC. Comparative study of perceived pain and nociceptive flexion reflex in man. Pain. (1977) 3:69-80.

40. Willer JC, Le Bars D, De Broucker T. Diffuse noxious inhibitory controls in man: Involvement of an opioidergic link. Eur J Pharmacol. (1990) 182:34755. doi: 10.1016/0014-2999(90)90293-F

41. Sandrini G, Arrigo A, Bono G, Nappi G. The nociceptive flexion reflex as a tool for exploring pain control systems in headache and other pain syndromes. Cephalalgia. (1993) 13:21-7.

42. Collins SL, Moore RA, McQuay HJ. The visual analogue pain intensity scale: what is moderate pain in millimetres? Pain. (1997) 72:95-7. doi: 10.1016/S0304-3959(97)00005-5

43. Bouhassira D, Attal N, Fermanian J, Alchaar H, Gautron M, Masquelier E, et al. Development and validation of the neuropathic pain symptom inventory. Pain. (2004) 108:248-57. doi: 10.1016/j.pain.2003.12.024

44. Cruccu G, Anand P, Attal N, Garcia-Larrea L, Haanpaa M, Jorum E, et al. EFNS guidelines on neuropathic pain assessment. Eur J Neurol. (2004) 11:153-62. doi: 10.1111/j.1468-1331.2004.00791.x

45. Desmeules JA, Cedraschi C, Rapiti E, Baumgartner E, Finckh A, Cohen P, et al. Neurophysiologic evidence for a central sensitization in patients with fibromyalgia. Arthritis Rheum. (2003) 48:1420-9. doi: 10.1002/art.10893

46. Banic B, Petersen-Felix S, Andersen OK, Radanov BP, Villiger PM, ArendtNielsen L, et al. Evidence for spinal cord hypersensitivity in chronic pain after whiplash injury and in fibromyalgia. Pain. (2004) 107:715. doi: 10.1016/j.pain.2003.05.001

47. De Broucker TH, Cesaro P, Willer JC, Le Bars D. Diffuse noxious inhibitory controls in man: involvement of the spinoreticular tract. Brain. (1990) 113:1223-34. doi: 10.1093/brain/113.4.1223

48. Langemark M, Bach FW, Jensen TS, Olesen J. Decreased nociceptive flexion reflex threshold in chronic tension-type headache. Arch Neurol. (1993) 50:1061-4. doi: 10.1001/archneur.1993.00540100056015

49. Sandrini G, Antonaci F, Lanfranchi S, Milanov I, Danilov A, Nappi G. Asymmetrical reduction of the nociceptive flexion reflex threshold in cluster headache. Cephalalgia. (2000) 20:64752. doi: 10.1111/j.1468-2982.2000.00096.x

50. Antonaci F, Sandrini G, Danilov A, Sand T. Neurophysiological studies in chronic paroxysmal hemicrania and hemicrania continua. Headache J Head Face Pain. (1994) 34:479-83. doi: 10.1111/j.1526-4610.1994.hed3408479.x

51. Brunoni AR, Nitsche MA, Bolognini N, Bikson M, Wagner T, Merabet $\mathrm{L}$, et al. Clinical research with transcranial direct current stimulation (tDCS): challenges and future directions. Brain Stimul. (2012) 5:17595. doi: 10.1016/j.brs.2011.03.002

52. McLaren ME, Nissim NR, Woods AJ. The effects of medication use in transcranial direct current stimulation: a brief review. Brain Stimul. (2018) 11:52-8. doi: 10.1016/j.brs.2017.10.006

53. Nitsche MA, Liebetanz D, Schlitterlau A, Henschke U, Fricke K, Frommann $\mathrm{K}$, et al. GABAergic modulation of DC stimulation-induced motor cortex excitability shifts in humans. Eur J Neurosci. (2004) 19:27206. doi: 10.1111/j.0953-816X.2004.03398.x

54. Liebetanz D, Nitsche MA, Tergau F, Paulus W. Pharmacological approach to the mechanisms of transcranial DC-stimulation-induced after-effects of human motor cortex excitability. Brain. (2002) 125:2238-47. doi: 10.1093/brain/awf238
55. Nitsche MA, Fricke K, Henschke U, Schlitterlau A, Liebetanz D, Lang N, et al. Pharmacological modulation of cortical excitability shifts induced by transcranial direct current stimulation in humans. J Physiol. (2003) 553:293301. doi: 10.1113/jphysiol.2003.049916

56. Rhudy JL, France CR. Defining the nociceptive flexion reflex (NFR) threshold in human participants: a comparison of different scoring criteria. Pain. (2007) 128:244-53. doi: 10.1016/j.pain.2006.09.024

57. Frahm Olsen M, Bjerre E, Hansen MD, Tendal B, Hilden J, Hróbjartsson A. Minimum clinically important differences in chronic pain vary considerably by baseline pain and methodological factors: systematic review of empirical studies. J Clin Epidemiol. (2018) 101:87-106.e2. doi: 10.1016/j.jclinepi.2018.05.007

58. Pacheco-Barrios K, Cardenas-Rojas A, Thibaut A, Costa B, Ferreira I, Caumo W, et al. Methods and strategies of tDCS for the treatment of pain: current status and future directions. Expert Rev Med Dev. (2020) 17:87998. doi: 10.1080/17434440.2020.1816168

59. Bushnell MC, Ceko M, Low LA. Cognitive and emotional control of pain and its disruption in chronic pain. Nat Rev Neurosci. (2013) 14:50211. doi: $10.1038 / \mathrm{nrn} 3516$

60. Truini A, Padua L, Biasiotta A, Caliandro P, Pazzaglia C, Galeotti $\mathrm{F}$, et al. Differential involvement of A-delta and A-beta fibres in neuropathic pain related to carpal tunnel syndrome. Pain. (2009) 145:1059. doi: 10.1016/j.pain.2009.05.023

61. Farina S, Valeriani M, Rosso T, Aglioti S, Tamburin S, Fiaschi A, et al. Transient inhibition of the human motor cortex by capsaicin-induced pain. A study with transcranial magnetic stimulation. Neurosci Lett. (2001) 314:97101. doi: 10.1016/s0304-3940(01)02297-2

62. Cogiamanian F, Ardolino G, Vergari M, Ferrucci R, Ciocca M, Scelzo E, et al. Transcutaneous spinal direct current stimulation. Front Psychiatry. (2012) 3:63. doi: 10.3389/fpsyt.2012.00063

63. Meyer-Frießem CH, Haag LM, Schmidt-Wilcke T, Magerl W, PogatzkiZahn EM, Tegenthoff M, et al. Transcutaneous spinal DC stimulation reduces pain sensitivity in humans. Neurosci Lett. (2015) 589:1538. doi: 10.1016/j.neulet.2015.01.029

64. Perrotta A, Bolla M, Anastasio MG, Serrao M, Sandrini G, Pierelli F. Modulation of temporal summation threshold of the nociceptive withdrawal reflex by transcutaneous spinal direct current stimulation in humans. Clin Neurophysiol. (2016) 127:755-61. doi: 10.1016/j.clinph.2015. 01.031

65. Parazzini M, Fiocchi S, Liorni I, Rossi E, Cogiamanian F, Vergari M, et al. Modeling the current density generated by transcutaneous spinal direct current stimulation (tsDCS). Clin Neurophysiol. (2014) 125:226070. doi: 10.1016/j.clinph.2014.02.027

66. Lenoir C, Jankovski A, Mouraux A. Anodal transcutaneous spinal direct current stimulation (tsDCS) selectively inhibits the synaptic efficacy of nociceptive transmission at spinal cord level. Neuroscience. (2018) 393:15063. doi: 10.1016/j.neuroscience.2018.10.007

Conflict of Interest: RF, MV, SB, and AP are founders and shareholders of Newronika Spa.

The remaining authors declare that the research was conducted in the absence of any commercial or financial relationships that could be construed as a potential conflict of interest.

Publisher's Note: All claims expressed in this article are solely those of the authors and do not necessarily represent those of their affiliated organizations, or those of the publisher, the editors and the reviewers. Any product that may be evaluated in this article, or claim that may be made by its manufacturer, is not guaranteed or endorsed by the publisher.

Copyright (c) 2021 Guidetti, Ferrucci, Vergari, Aglieco, Naci, Versace, PachecoBarrios, Giannoni-Luza, Barbieri, Priori and Bocci. This is an open-access article distributed under the terms of the Creative Commons Attribution License (CC BY). The use, distribution or reproduction in other forums is permitted, provided the original author(s) and the copyright owner(s) are credited and that the original publication in this journal is cited, in accordance with accepted academic practice. No use, distribution or reproduction is permitted which does not comply with these terms. 